\title{
Analysis of Electroencephalographic Dynamic Functional Connectivity in Alzheimer's Disease
}

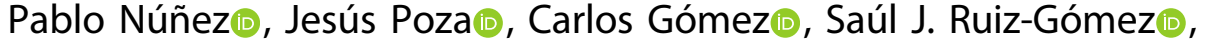 \\ Víctor Rodríguez-González, Miguel Ángel Tola-Arribas, Mónica Cano, \\ and Roberto Hornero
}

\begin{abstract}
The aim of this study was to characterize the dynamic functional connectivity of resting-state electroencephalographic (EEG) activity in Alzheimer's disease (AD). The magnitude squared coherence $(\mathrm{MSCOH})$ of 50 patients with dementia due to $\mathrm{AD}$ and 28 cognitively healthy controls was computed. $\mathrm{MSCOH}$ was estimated in epochs of $60 \mathrm{~s}$ subdivided in overlapping windows of different lengths $(1,2,3,5$ and $10 \mathrm{~s} ; 50 \%$ overlap). The effect of epoch length was tested on $\mathrm{MSCOH}$ and it was found that $\mathrm{MSCOH}$ stabilized at a window length of $3 \mathrm{~s}$. We tested whether the $\mathrm{MSCOH}$ fluctuations observed reflected actual changes in functional connectivity by means of surrogate data testing, with the standard deviation of $M S C O H$ chosen as the test statistic. The results showed that the variability of the measure could be due to dynamic functional connectivity. Furthermore, a significant reduction in the dynamic $\mathrm{MSCOH}$ connectivity of AD patients compared to controls was found in the delta $(0-4 \mathrm{~Hz})$ and beta-1 $(13-30 \mathrm{~Hz})$ bands. This indicated that $\mathrm{AD}$ patients show lesser variation in neural connectivity during resting state. Finally, a correlation between relative power and standard deviation was found, suggesting that an increase/peak in power spectrum could be a pre-requisite for dynamic functional connectivity in a specific frequency band.
\end{abstract}

P. Núñez $(\bowtie) \cdot J$. Poza · C. Gómez · S. J. Ruiz-Gómez

V. Rodríguez-González $\cdot$ R. Hornero

Biomedical Engineering Group, University of Valladolid,

Valladolid, Spain

e-mail: pablo.nunez@gib.tel.uva.es

M. Á. Tola-Arribas

Neurology Service, Río Hortega University Hospital, Valladolid, Spain

M. Cano

Clinical Neurophysiology Service, Río Hortega University

Hospital, Valladolid, Spain

\section{Keywords}

Alzheimer's disease - Dynamic functional connectivity Electroencephalogram $\bullet$ Neural dynamics

Coherence $\cdot$ Relative power

\section{Introduction}

Alzheimer's disease (AD) neurodegeneration has an effect on the temporally coordinated brain networks, which underlie cognitive functions. These networks become more abnormal with the progression of $\mathrm{AD}$, as alterations in the processing and transmission of information begin to appear [1]. The aberrant brain networks associated to $\mathrm{AD}$ can be reflected in the alterations of the synchronization patterns and brain connectivity observed in resting-state electroencephalographic (EEG) recordings [1].

The majority of studies focusing on brain synchronization and connectivity during resting state assume that functional connectivity (FC) remains temporally stationary. However, it is important to take the spontaneous fluctuations of brain activity into account, as it has been suggested that resting-state activity is not stationary [2]. Thus, the quantification of dynamic changes in FC metrics could provide relevant information regarding the stability of brain networks [3].

Most dynamic functional connectivity (dFC) studies in the literature have been performed with functional magnetic resonance imaging (fMRI) recordings [3]. Therefore, the study of dFC on electroencephalographic (EEG) recordings is of great interest. In the specific case of $\mathrm{AD}$, only a small number of studies have addressed the characterization of FC variability patterns [3].

This paper presents a novel methodology, aimed at obtaining a first approximation to the dynamics of $\mathrm{FC}$ in $\mathrm{AD}$. Specifically, this study addresses the following research questions: (i) what epoch length is needed to obtain stable connectivity measures?; (ii) can $\mathrm{dFC}$ be found in $\mathrm{AD}$ 
patients and healthy controls?; (iii) do dFC patterns differ between both groups?

\section{Materials}

\subsection{Subjects}

A population of 28 healthy control subjects (C) and 50 patients with AD was analyzed. The subjects were matched by age. Patients were diagnosed according to the criteria of the National Institute on Aging and Alzheimer's Association (NIA-AA) [4]. The controls were elderly people without cognitive impairment, or history of neurological or psychiatric diseases. None of the participants took medications that could influence EEG records.

The socio-demographic characteristics of each group are specified in Table 1. All participants and caregivers were informed about the research and study protocol and gave their written and informed consent. The Ethical Committee of the "Río Hortega" University Hospital (Valladolid, Spain) approved the study according to the Code of Ethics of the World Medical Association (Declaration of Helsinki).

\subsection{Electroencephalographic Recordings}

EEG signals were recorded using a 19-channel EEG system (XLTEK $^{\circledR}$, Natus Medical) at the Department of Clinical Neurophysiology of the "Río Hortega" University Hospital. EEG activity was acquired from Fp1, Fp2, Fz, F3, F4, F7, F8, Cz, C3, C4, T3, T4, T5, T6, Pz, P3, P4, O1 y O2, at a sampling frequency of $200 \mathrm{~Hz}$. Subjects were asked to remain with eyes closed, awake, and still during EEG acquisition. Five minutes of EEG activity were recorded for each subject. After a preliminary independent component analysis to remove artifacted components, the EEG recordings were then preprocessed in three steps: (i) filtering using a notch filter $(50 \mathrm{~Hz})$ and a Hamming window bandpass filter ([1 70] Hz); (ii) segmentation into $5 \mathrm{~s}$ epochs; and (iii) visual rejection of artifacts, selecting the first 60 consecutive seconds without artifacts for each subject.

\section{Methods}

\subsection{Estimation of Variability}

Coherency $(\mathrm{COH})$ is a measure that analyzes the consistency between the EEG activity of different pairs of electrodes in order to characterize the connectivity between brain regions. $\mathrm{COH}$ is the standardized cross-spectrum of signals $X$ and $Y$ across trials, divided by the product of their power spectrum. Magnitude squared $\mathrm{COH}(\mathrm{MSCOH})$ combines sensitivity to both phase and magnitude synchrony and is defined as [5]:

$$
\operatorname{MSCOH}_{x y}(f, t)=\left|\operatorname{COH}_{x y}(f, t)\right|^{2}=\frac{\left|S_{X Y}(f, t)\right|^{2}}{P_{X}(f, t) P_{y}(f, t)}
$$

where $S_{X Y}$ is the cross-spectrum of $X$ and $Y$, and $P_{X}$ and $P_{Y}$ are the power spectral density (PSD) of $X$ and $Y$, respectively. The relative power (RP) was computed from the PSD in the conventional frequency bands: delta $(\delta, 1-4 \mathrm{~Hz})$, theta $(\theta, 4-8 \mathrm{~Hz})$, alpha $(\alpha, 8-13 \mathrm{~Hz})$, beta-1 $(\beta 1,13-19 \mathrm{~Hz})$, beta-2 $(\beta 2,19-30 \mathrm{~Hz})$ and gamma $(\gamma, 30-70 \mathrm{~Hz})$.

\subsection{Protocol}

In order to study the dynamic properties of functional connectivity coupling patterns, $\mathrm{MSCOH}$ was computed between each pair of electrodes over the $60 \mathrm{~s}$ epochs by means of sliding windows with $50 \%$ overlap. Afterwards, the mean value of $\mathrm{MSCOH}\left(\mu_{\mathrm{MSCOH}}\right)$ and the standard deviation $\left(\kappa_{\mathrm{MSCOH}}\right)$ were obtained. $\kappa_{\mathrm{MSCOH}}$ was used as the test statistic in order to detect the existence of dFC [6]. The connectivity matrixes for each frequency band were grouped into five regions (frontal, left-temporal, right-temporal, central and parieto-occipital) and inter-regional and intra-regional $\mu_{\mathrm{MSCOH}}$ and $\kappa_{\mathrm{MSCOH}}$ values were averaged among the electrodes within each region pair. After this procedure, the connectivity matrix was reduced to a $5 \times 5$ size (5 regions). All subsequent analyses were performed on these matrixes. This procedure was performed on windows of $1,2,3,5$ and $10 \mathrm{~s}$, in the aforementioned 6 conventional frequency bands.

Table 1 Socio-demographic and clinical data. Mean values \pm standard deviation. A: primary education or below; B: secondary education or above; MMSE: Mini-Mental State Examination

\begin{tabular}{l|l|l}
\hline Data & Alzheimer's disease & Controls \\
\hline Number of subjects & 50 & 28 \\
\hline Age (years) & $79.9 \pm 5.8$ & $76.1 \pm 34.0$ \\
\hline Gender (male:female) & $21: 28$ & $8: 20$ \\
\hline Education level (A:B) & $37: 13$ & $9: 19$ \\
\hline MMSE & $21.2 \pm 4.0$ & $28.9 \pm 1.1$
\end{tabular}




\subsection{Statistical Analysis}

Shapiro-Wilk and Levene tests showed that $\mu_{\mathrm{MSCOH}}$ and $\kappa_{\mathrm{MSCOH}}$ values did not meet parametric test conditions. Then, the Mann-Whitney $U$-test was used to evaluate the differences between groups across each frequency band and region. An FDR correction was performed in order to correct for multiple comparisons. Furthermore, a Spearman correlation analysis was performed between the RP values and the average $\kappa_{\mathrm{MSCOH}}$ values of each channel.

\subsection{Analysis of Window Stability}

The Friedman test was used on the $\mu_{M S C O H}$ values to detect the effects of window length on the MSCOH measures. In case the Friedman test showed a significant effect, Dunn's multiple comparison test was applied to determine the window in which the MSCOH measurements became stable, defined as the shortest window length that does not show significant differences with longer window sizes [7].

\subsection{Detection of Dynamic Functional Connectivity}

It is important to take into account the fact that the mere presence of fluctuations in connectivity measures is not sufficient proof of the existence of dFC. Due to the noisy nature of the recordings, the fact that the observed connectivity values are estimates of the true FC values cannot be ignored [6]. Therefore, in order to determine whether the observed fluctuations reflect real FC changes, an adequate statistical test must be carried out [6]. We followed the statistical test described by Prichard and Theiler [8], which has been previously used by Hindriks et al. [6]. In our case, 1000 surrogate versions of each EEG segment were constructed from the original signals.

\section{Results and Discussion}

First, we determined the window size in which the MSCOH measurements became stable. It was found that for both groups this size was $3 \mathrm{~s}$. All further tests were thus performed on values obtained with a $3 \mathrm{~s}$ sliding window. We then assessed whether the $\kappa_{M S C O H}$ values were statistically significant [6]. For each, inter-regional and intra-regional pair we performed an FDR correction on the $p$-values associated with the $z$-scores of all the connections within each pair. After this, we determined that a regional pair had $\mathrm{dFC}$ if at least one of the connections within it had a statistically significant $\kappa_{\mathrm{MSCOH}}$ value after the FDR correction.

Figure 1a shows the number of subjects that showed statistically significant $\mu_{\mathrm{MSCOH}}$ in each regional pair. The beta-1 band showed the highest number of regional pairs

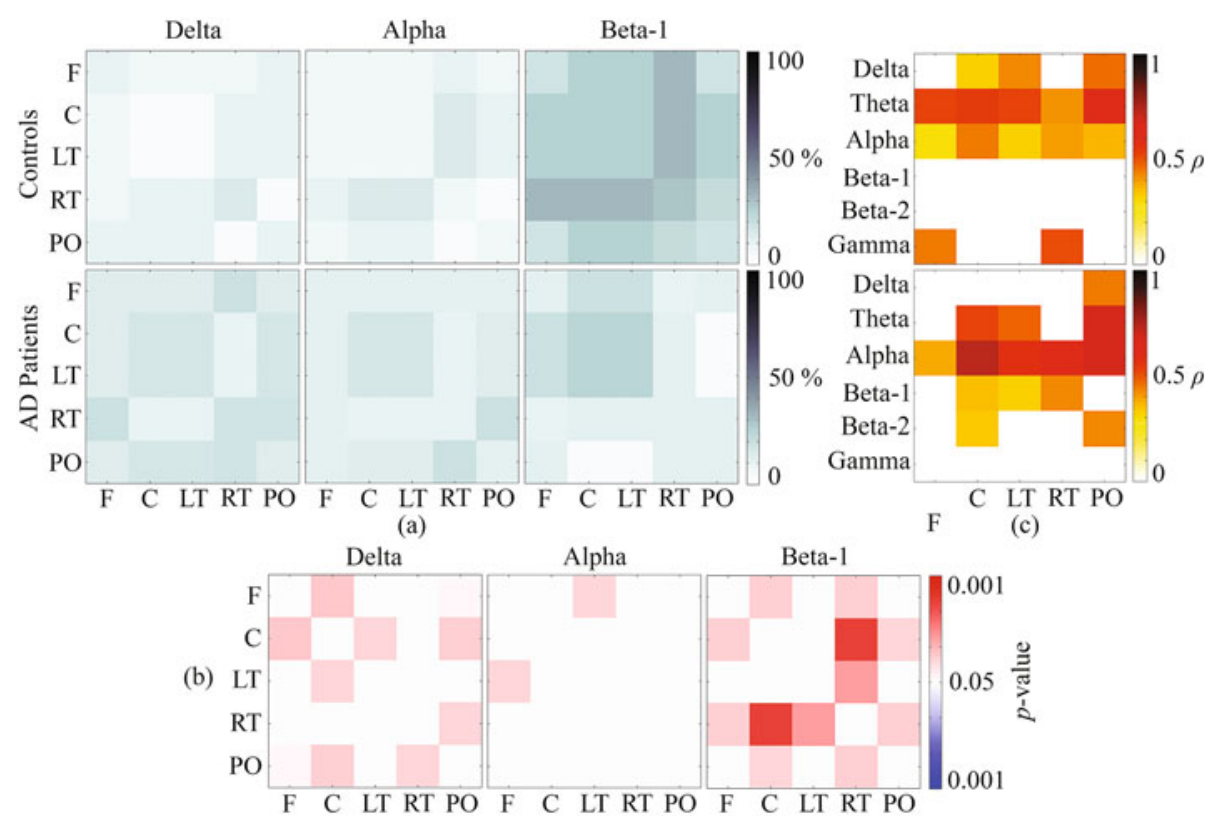

Fig. $1 \mathrm{MSCOH} \mathrm{dFC}$ analysis for a 3-s sliding window. a Percentage of subjects that showed statistically significant $\mu_{\mathrm{MSCOH}}$ in each regional pair for each group. b Statistically significant $p$-values for the $\kappa_{\mathrm{MSCOH}}$ comparisons between groups. Red values indicate greater $\kappa_{M S C O H}$ for C than $\mathrm{AD}$ patients, while blue values indicate greater $\kappa_{M S C O H}$ for $\mathrm{AD}$ patients than C. c Correlation between RP values and $\kappa_{M S C O H}$ for each group. Only the bands with significant between-group differences are shown. Correspondence with regions F: frontal, C: central, LT: left-temporal, RT: right-temporal, PO: parieto-occipital (Color figure online) 
with dFC, especially for controls, nearing $50 \%$ of subjects in some cases. The statistical differences in $\mu_{\mathrm{MSCOH}}$ between groups are also shown in Fig. 1b. The most statistically significant differences were located in the beta-1 band, especially in the connections between the right-temporal region and the remaining ones. These results are consistent with previous findings that support the role of the right hemisphere in brain disconnection related to $\mathrm{AD}$ [9]. Less statistically significant differences were also found in the delta band. Controls showed more variation in connectivity than $\mathrm{AD}$ patients, which is in agreement with other studies that found a loss of irregularity and variability in AD neural activity [9].

The correlation analysis between RP and the average $\kappa_{M S C O H}$ values of each EEG channel in each band, displayed in Fig. 1c, showed statistically significant positive correlation in the delta, theta and alpha band for most regions in controls. AD patients, on the other hand, showed weaker positive correlation in the beta-1 band as well, suggesting that spectral power is a pre-requisite for $\mathrm{dFC}$.

This study has some limitations. Firstly, more controls should be included in the database in order to balance the number of $\mathrm{AD}$ patients and controls. Furthermore, a third group of mild cognitive impairment (MCI) patients should be included in future studies, given its importance as a prodromal form of AD [9].

\section{Conclusion}

The results suggest that MSCOH variability could be due to dFC. Moreover, AD patients show lesser variation in neural connectivity than controls, which suggests a loss of variability in AD. Finally, the correlation between relative power and $\kappa_{\mathrm{MSCOH}}$ hints that a peak in power spectrum in a frequency band could be a pre-requisite for $\mathrm{dFC}$.
Acknowledgements This study has been partially funded by projects TEC2014-53196-R of 'Ministerio de Economía y Competitividad' and FEDER, the project 'Análisis y correlación entre el genoma completo y la actividad cerebral para la ayuda en el diagnóstico de la enfermedad de Alzheimer' (Inter-regional cooperation program VA Spain-Portugal POCTEP 2014-2020) of the European Commission and FEDER, and project VA037U16 of the 'Junta de Castilla y León and FEDER. P. Núñez and S. J. Ruiz are in receipt of predoctoral grants co-financed by the 'Junta de Castilla y León' and ESF.

Conflict of Interest There are no conflicts of interest that could influence this research work.

\section{References}

1. Babiloni $\mathrm{C}$, et al.: Brain neural synchronization and functional coupling in Alzheimer's disease as revealed by resting state EEG rhythms. Int J Psychophysiol 103, 88-102 (2015).

2. Hansen ECA, et al.: Functional connectivity dynamics: Modeling the switching behavior of the resting state. Neuroimage 105, 525535 (2015)

3. Hutchison RM, et al.: Dynamic functional connectivity: Promise, issues, and interpretations. Neuroimage 80, 360-378 (2013).

4. McKhann G, et al.: The diagnosis of dementia due to Alzheimer's disease: Recommendations from the National Institute on AgingAlzheimer's Association workgroups on diagnostic guidelines for Alzheimer's disease. Alzheimers Dement 7(3), 263-26 (2011).

5. Roach BJ, Mathalon DH.: Event-related EEG time-frequency analysis: An overview of measures and an analysis of early gamma band phase locking in schizophrenia. Schizophr Bull 34(5), 907926 (2008).

6. Hindriks R, et al.: Can sliding-window correlations reveal dynamic functional connectivity in resting-state fMRI? Neuroimage 127, 242-256 (2016).

7. Fraschini M, et al.: The effect of epoch length on estimated EEG functional connectivity and brain network organisation. J Neural Eng 13(3), 36015 (2016).

8. Prichard D, Theiler J.: Generating surrogate data for time series with several simultaneously measured variables. Phys Rev Lett 73(7), 951-954 (1994).

9. Poza J, et al.: Spatio-Temporal fluctuations of neural dynamics in mild cognitive impairment and Alzheimer's disease. Curr Alzheimer Res 14(9), 924-936 (2017). 